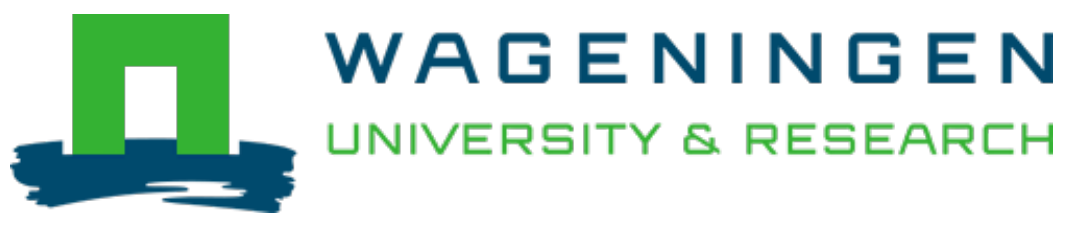

\author{
Wrong place, wrong time: climate change-induced range shift across fragmented \\ habitat causes maladaptation and declined population size in a modelled bird \\ species \\ Global Change Biology \\ Cobben, M.M.P.; Verboom, J.; Opdam, P.F.M.; Hoekstra, R.F.; Jochem, R. et al \\ https://doi.org/10.1111/j.1365-2486.2012.02711.x
}

This article is made publicly available in the institutional repository of Wageningen University and Research, under the terms of article $25 \mathrm{fa}$ of the Dutch Copyright Act, also known as the Amendment Taverne. This has been done with explicit consent by the author.

Article 25 fa states that the author of a short scientific work funded either wholly or partially by Dutch public funds is entitled to make that work publicly available for no consideration following a reasonable period of time after the work was first published, provided that clear reference is made to the source of the first publication of the work.

This publication is distributed under The Association of Universities in the Netherlands (VSNU) 'Article $25 \mathrm{fa}$ implementation' project. In this project research outputs of researchers employed by Dutch Universities that comply with the legal requirements of Article 25fa of the Dutch Copyright Act are distributed online and free of cost or other barriers in institutional repositories. Research outputs are distributed six months after their first online publication in the original published version and with proper attribution to the source of the original publication.

You are permitted to download and use the publication for personal purposes. All rights remain with the author(s) and / or copyright owner(s) of this work. Any use of the publication or parts of it other than authorised under article $25 \mathrm{fa}$ of the Dutch Copyright act is prohibited. Wageningen University \& Research and the author(s) of this publication shall not be held responsible or liable for any damages resulting from your (re)use of this publication.

For questions regarding the public availability of this article please contact openscience.library@wur.nl 


\title{
Wrong place, wrong time: climate change-induced range shift across fragmented habitat causes maladaptation and declined population size in a modelled bird species
}

\author{
MARLEEN M. P. COBBEN*†, JANA VERBOOM†, PAUL F. M. OPDAM $†$, \\ ROLF F. HOEKSTRA $\S$, RENÉ JOCHEM† and MARINUS J. M. SMULDERS† \\ *Plant Research International, Wageningen UR Plant Breeding, PO Box 16, 6700 AA, Wageningen, The Netherlands, $\uparrow$ Alterra, \\ Wageningen UR, Wageningen, The Netherlands, \$Land Use Planning Group, Wageningen UR, Wageningen, The Netherlands, \\ $\S$ Laboratory of Genetics, Wageningen UR, Wageningen, The Netherlands
}

\begin{abstract}
Many species are locally adapted to decreased habitat quality at their range margins, and therefore show genetic differences throughout their ranges. Under contemporary climate change, range shifts may affect evolutionary processes at the expanding range margin due to founder events. In addition, populations that are affected by such founder events will, in the course of time, become located in the range centre. Recent studies investigated evolutionary changes at the expanding range margin, but have not assessed eventual effects across the species' range. We explored the possible influence of range shift on the level of adaptation throughout the species' total range. For this we used a spatially explicit, individual-based simulation model of a woodland bird, parameterized after the middle spotted woodpecker (Dendrocopos medius) in fragmented habitat. We simulated its range under climate change, and incorporated genetic differences at a single locus that determined the individual's degree of adaptation to optimal temperature conditions. Generalist individuals had a large thermal tolerance, but relatively low overall fitness, whereas climate specialists had high fitness combined with a small thermal tolerance. In equilibrium, the populations in the range centre were comprised of the specialists, whereas the generalists dominated the margins. In contrast, under temperature increase, the generalist numbers increased at the expanding margin and eventually also occupied the centre of the shifting range, whereas the specialists were located in the retracting margins. This was caused by founder events and led to overall maladaptation of the species, which resulted in a reduced metapopulation size and thus impeded the species' persistence. We therefore found no evidence for a complementary effect of local adaptation and range shifts on species' survival. Instead, we showed that founder events can cause local maladaptation which can amplify throughout the species' range, and, as such, hamper the species' persistence under climate change.
\end{abstract}

Keywords: climate change, Dendrocopos medius, founder effect, habitat fragmentation, maladaptation, range shift, simulation model

Received 14 November 2011 and accepted 18 March 2012

\section{Introduction}

The genetic composition of a population of a natural species results from the interaction of population demography and adaptation to environmental conditions. However, conditions across a species' biogeographical range vary, many species show genetic differences when comparing central and marginal populations (Sexton et al., 2009). Populations in species' range margins have been shown to adapt to average lower habitat quality (Kawecki, 2008).

Under contemporary climate change (IPCC, 2007) many species have been observed to shift their ranges corresponding to the increased temperature (Chen et al.,

Correspondence: Marleen M. P. Cobben, tel. +31 317481 082, e-mail: marleen.cobben@wur.nl
2011; Parmesan \& Yohe, 2003; Root et al., 2003). Range shifts may have genetic consequences, such as founder events which occur at the expanding range margins (Garroway et al., 2011; Hill et al., 2011; Lee, 2002; Mayr, 1942). Founder events can affect local evolutionary processes (Excoffier et al., 2009; Travis et al., 2007; Vila et al., 2003), and have been observed to generate signatures of reduced neutral allelic variation in recently established populations (Excoffier et al., 2009; Hewitt, 1996; Hill et al., 2011). Therefore, contemporary climate change may affect the frequencies of genotypes in populations at species' range margins. Furthermore, under range shift, the locations of these populations, originally at the range margin, will shift closer to the species' range centre, whereas the populations at the retracting range margin become extinct. Combined with the observed persistence of the founder effect (Hewitt, 1996), this 
may eventually influence the allele frequencies across the entire species' range (Cobben et al., 2011; Hewitt, 1996; McInerny et al., 2009), and therefore the overall degree of adaptation to local conditions. As this may result in local maladaptation and, consequently, decreased population sizes and persistence, it is of primary importance to understand how species' responses to climate change interact with the heterogeneous genetic structure throughout their ranges.

Changes in local adaptive genetic diversity under climate change have mainly been investigated in the expanding species' range margins and range interiors (Excoffier et al., 2009; Hill et al., 2011, 1999; Parmesan, 2006; Thomas et al., 2001), with some research effort for the retracting range margins (Hampe \& Petit, 2005; Jump et al., 2006). Range-wide assessments exist mostly in modelling studies of neutral allelic diversity (Cobben et al., 2011; McInerny et al., 2009). However, for past climate changes, founder events were shown to affect the genetic structure of populations in large parts of species' ranges (Hewitt, 1996). Contemporary habitat fragmentation is furthermore likely to increase the occurrences and consequences of founder events upon range expansions, as can be deduced from theory (Mayr, 1942) and simulations (Cobben et al., 2012; Edmonds et al., 2004; Klopfstein et al., 2006).

Therefore, we investigated the interaction of local adaptation and founder events across a species' total range in fragmented habitat under climate change. We simulated how a genetic difference in adaptation between central and marginal populations in a species' range along a temperature gradient in spatially structured habitat is affected by climate change, allowing for range shifts and local adaptation. For this we used a metapopulation based on ecological information of the middle spotted woodpecker (Dendrocopos medius), in which a single gene determined the degree of adaptation to local temperature conditions. Various climate change scenarios, including both temperature increase and increased weather variability (Cobben et al., 2011; IPCC, 2007; Schippers et al., 2011) were applied. We specifically asked:

- how the local frequencies of the different genotypes in the species' range changed under range shifts,

- how the changed distribution affected the persistence of the species and

- whether or not, and if so, when the original distribution of the various genotypes recovered after stabilization of the temperature.

\section{Materials and methods}

For this study we used METAPHOR, a simulation model for metapopulation demography (Verboom et al., 2001; Vos et al.,
2001). The model has been extended to allow for stochastic temperature increase by Schippers et al. (2011). We further extended the model with a genetic module for adaptive traits. Reproduction, dispersal and survival were based on population density and habitat quality. Habitat quality was solely dependent on the time- and location-specific temperature. The individual's perception of the habitat quality depended on its genotype. As such we could simulate the effect of stochastic temperature increase in the spatial distribution of the different genotypes. For detailed information see the online Supporting Information. Table 1 gives an overview of all species, genetic and climate parameters used.

We modelled a woodland bird, parameterized as the middle spotted woodpecker Dendrocopos medius. This species is a moderate disperser, yet not affected by infrastructural and urban barriers. It also is fairly specific in its habitat choice and has limited population growth capacity. It therefore represents a species in which the habitat distribution is fragmented over large areas, and with a population demography which makes it sensitive to range shifts.

Under temperature increase, the temperature optimum was shifting northwards across the species' range (see below and Table 1). Temperature suitability was translated to a habitat patch quality ranging from 0 to 1 , based on distance from the climate optimum and on the half value parameter used, following a Gaussian curve (Schippers et al., 2011). We used three temperature increase scenarios, based on work by the Hadleycentre (2003). The first scenario was a temperature increase of $1{ }^{\circ} \mathrm{C}$ from year 2000 to 2100 . As far as we currently know, this is an unrealistically small temperature increase prediction for the coming century, but it was incorporated as an absolute minimal change to compare with the more likely scenarios of a $2{ }^{\circ} \mathrm{C}$ and $4{ }^{\circ} \mathrm{C}$ temperature increase by 2100 (Hadleycentre, 2003), which are based, respectively, on IPCC SRES emission scenarios B1 and A2. When translating these scenarios to temperature isocline shift rates, we assumed that the simulated metapopulation was situated along the European Atlantic coast. For the SRES A1B scenario, the Hadleycentre (2003) predicts no regional differences in change of temperature within this stretch of coast for the year 2080. We therefore assumed a similar rate of temperature increase across the simulated landscape. Unaffected by mountain ranges, this specific coast line has a temperature gradient of $0.0042{ }^{\circ} \mathrm{C}$ per $\mathrm{km}$ (Schippers et al., 2011). The current average standard deviation of the average temperature in this region is $0.59{ }^{\circ} \mathrm{C}$ (Schippers et al., 2011). We used the temperature gradient to convert the explored temperature increase rates and the yearly temperature variability in ${ }^{\circ} \mathrm{C}$ per year and ${ }^{\circ} \mathrm{C}$, respectively, to geographical distances (per year) (see the Supporting Information). The three temperature increase scenarios as such resulted in equivalent isocline shift rates of 2, 4 and $8 \mathrm{~km}$ per year, respectively, with a standard deviation of $140 \mathrm{~km}$. To assess the effect of temperature variability, we also applied standard deviation levels of $280 \mathrm{~km}$ and $0 \mathrm{~km}$.

We allowed two different alleles at the adaptive locus, the climate specialist allele $S$ and the climate generalist allele $G$. The alleles could mutate with frequency $10^{-6}$ (Nachman \& 
Table 1 Model parameters used. Species and climate parameters are those used by Schippers et al. (2011). Parameter names link this table to the functions in the Detailed model description (online Supporting Information)

\begin{tabular}{|c|c|c|c|}
\hline Parameter description & Value & Unit & Parameter name \\
\hline \multicolumn{4}{|l|}{ Landscape parameters } \\
\hline number of patches & 3000 & & \\
\hline patch area & 50 & ha & \\
\hline patch carrying capacity & 20 & individuals & \\
\hline \multicolumn{4}{|l|}{ Species parameters } \\
\hline perceived habitat quality genotype SS & 1 & & HQfactor $_{\text {gen }}$ \\
\hline perceived habitat quality genotype SG & 0.95 & & $H_{Q}$ factor $_{\text {gen }}$ \\
\hline perceived habitat quality genotype GG & 0.9 & & $H_{Q}$ factor $_{\text {gen }}$ \\
\hline \multicolumn{4}{|l|}{ Recruitment } \\
\hline area per reproductive unit & 5 & ha & \\
\hline recruitment at density $=0$ and quality $=1$ & 2.4 & juveniles/female & $N_{O_{-} P D O H Q 1}$ \\
\hline recruitment at density $=1$ and quality $=1$ & 1.8 & juveniles/female & $N_{O_{-} P D 1 H Q 1}$ \\
\hline recruitment at density $=0$ and quality $=0$ & 0 & juveniles/female & $N_{O_{-} P D O H Q O}$ \\
\hline \multicolumn{4}{|l|}{ Dispersal } \\
\hline maximum dispersal distance & 15 & $\mathrm{~km}$ & \\
\hline $\begin{array}{l}\text { maximum detection distance } \\
\text { juvenile dispersal probability }\end{array}$ & 150 & $\mathrm{~m}$ & $l$ \\
\hline at density $=0$ and quality $=1$ & 0 & year $^{-1}$ & $P_{D P D O H Q 1}$ \\
\hline at density $=1$ and quality $=1$ & 0.6 & year $^{-1}$ & $P_{D P D 1 H Q 1}$ \\
\hline at density $=0$ and quality $=0$ & 1 & year $^{-1}$ & $P_{D_{-} P D 1 H Q 0}$ \\
\hline adult dispersal probability & & & \\
\hline at density $=0$ and quality $=1$ & 0 & year $^{-1}$ & $P_{D_{-} P D 0 H Q 1}$ \\
\hline at density $=1$ and quality $=1$ & 0.1 & year $^{-1}$ & $P_{D_{-} P D 1 H Q 1}$ \\
\hline at density $=0$ and quality $=0$ & 0.5 & year $^{-1}$ & $P_{D_{-} P D 1 H Q 0}$ \\
\hline \multicolumn{4}{|l|}{ Survival } \\
\hline juvenile survival probability & 1 & year $^{-1}$ & $P_{S_{-} P D 0 H Q 1}, P_{S_{-} P D 1 H Q 1}, P_{S_{-} P D O H Q O}$ \\
\hline adult survival probability at quality $=1$ & 0.8 & year $^{-1}$ & $P_{S_{-} P D O H Q 1}, P_{S_{-} P D 1 H Q 1}$ \\
\hline adult survival probability at quality $=0$ & 0.55 & year $^{-1}$ & $P_{S_{-} P D O H Q O}$ \\
\hline \multicolumn{4}{|l|}{ Genetic parameters } \\
\hline allele range at initialization & S and $G$ & & \\
\hline allele range after mutation & S and $G$ & & \\
\hline mutation rate & $10^{-6}$ & generation $^{-1}$ & \\
\hline \multicolumn{4}{|l|}{ Climate parameters } \\
\hline temperature isocline speed & $2,4,8$ & $\mathrm{~km}_{\text {year }}{ }^{-1}$ & $T$ \\
\hline weather variability & $0,140,280$ & $\mathrm{~km}$ & $\sigma_{d}$ \\
\hline temperature tolerance genotype SS & 600 & $\mathrm{~km}$ & $H_{g e n}$ \\
\hline temperature tolerance genotype SG & 700 & $\mathrm{~km}$ & $H_{\text {gen }}$ \\
\hline temperature tolerance genotype GG & 800 & $\mathrm{~km}$ & $H_{g e n}$ \\
\hline initial temperature optimum location & 400 & $\mathrm{~km}$ from the south edge & $Y_{o p t, 0}^{o}$ \\
\hline \multicolumn{4}{|l|}{ Model run parameters } \\
\hline burn-in & 3000 & years & \\
\hline investigated time points after burn-in & $\begin{array}{c}0,25,50,75,100 \\
125,150,200,250 \\
300,400,500,600\end{array}$ & years & \\
\hline
\end{tabular}

Crowell, 2000). Note that we use the terms specialist and generalist for the individuals carrying these different alleles, analogous to the use of these terms for different species, but here it concerns different genotypes within one species. Our species was diploid, leading to the three potential genotypes SS, SG and GG. The climate generalists, genotype GG, had a relatively large thermal tolerance, but, as a trade-off, a lower over- all fitness (Fig. 1). The individuals' fitness was determined by local habitat quality, based on local and temporal temperature, as explained above. For the generalists, we therefore restrained the perceived habitat quality, applying a fitness factor of 0.9 . The climate specialists with the SS genotype had a fitness factor of one, and thus no restrained habitat quality, but a relatively small thermal tolerance (Fig. 1). The individuals 


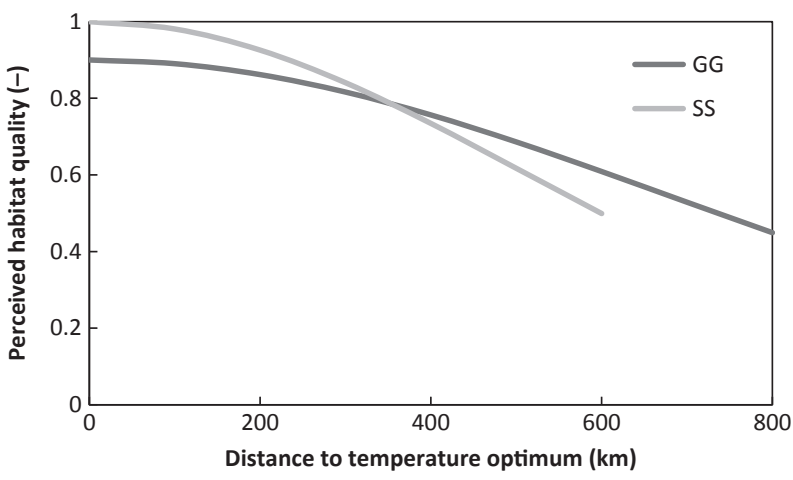

Fig. 1 The perceived habitat quality, equivalent to relative fitness as a function of the individual's genotype and its distance to the temperature optimum. The GG-individuals are climate generalists, and the SS-individuals climate specialists.

with the SG-genotype were intermediate in both thermal tolerance and fitness. The perceived habitat quality and the population density determined the survival rate, the number of offspring and the dispersal rate (online Supporting Information). As such we created a metapopulation that, under equilibrium conditions, had an increasing dispersal rate, and decreasing survival and recruitment rates from the centre to the range margin.

\section{Simulation experiments}

1 To study how the local frequencies of the different genotypes in the species' range changed under climate change, we investigated the spatial distributions of the SS, SG and GG genotypes throughout time under nine climate change scenarios. For these scenarios we combined the three different rates of temperature isocline shift of 2, 4 and $8 \mathrm{~km}$ year $^{-1}$ with the three standard deviations 0,140 and $280 \mathrm{~km}$. The temperature isoclines were simulated to move northward for 600 years. We additionally assessed the levels of neutral allelic diversity to check for the occurrence of founder events.

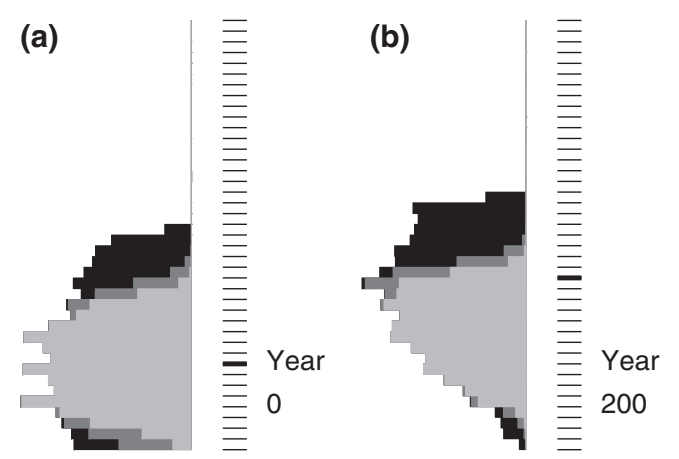

2 To investigate how the changed genotype distributions affected the persistence of the species, we simulated the metapopulation, subjected to the three rates of temperature increase and the standard variation of $140 \mathrm{~km}$, under two additional parameter settings. In these settings, the metapopulation contained only one allele at the adaptation locus, so it had either only individuals of the SS genotype or of the GG genotype. We then assessed the size of the metapopulation under all three different parameter sets, so while containing only allele $S$, only allele $G$ or both alleles $S$ and $\mathrm{G}$, as in experiment 1 .

3 To investigate the recovery of the original genotype distribution after stabilization of the temperature, we subjected the metapopulation to the three temperature increase scenarios, which were stopped after 50 years or multiples of 50 years up to 700 years. The model was run for another 300 years after the stop with stabilized temperature, while documenting the genotype distributions.

\section{Results}

\section{Effects of climate change on the spatial distribution of the genotypes}

The original distribution of the genotypes in the metapopulation was changed under the range shift that was caused by the temperature increase (Fig. 2). This led to an increase in the relative number of generalists (GG) and the relative size of the area in which they lived. This increase occurred at the cost of the specialist (SS) and intermediate (SG) individuals and eventually resulted in the extinction of the specialist allele $S$ in the metapopulation. We also observed that after a period of temperature increase, the relative positions of the SS and GG individuals to the temperature optimum was inverse in comparison to the positions in the initial distribution (compare in Fig. 2 the years 400 and 600 with year 0 ). The generalists occurred at the temperature optimum, where the
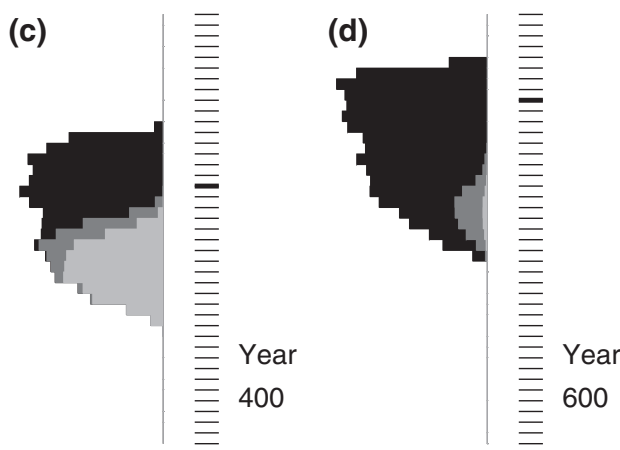

Fig. 2 The distributions and numbers of the GG (black bars), SG (dark grey bars) and SS (light grey bars) genotypes in time through the model space under the temperature isocline shift rate of $2 \mathrm{~km} \mathrm{year}^{-1}$, with standard deviation of $140 \mathrm{~km}$. The lengths of the bars indicate the sum of the local numbers of individuals. The thin horizontal lines represent the model space, cut into ranges of $50 \mathrm{~km}$. The bold black line in the model space indicates the location of the average temperature optimum along the total range of $2000 \mathrm{~km}$ in time, which is indicated in the lower right corners of each of the figures. 
specialists have a selective advantage, whereas the specialists lived at the range margin, where the generalists have a higher fitness. Both the effects of inverse positioning and relative increase in generalists were seen under all temperature increase scenarios. The metapopulation only survived under the isocline shift rate of $2 \mathrm{~km}$ year $^{-1}$. Under isocline shift rates of $4 \mathrm{~km}_{\text {year }}{ }^{-1}$ and 8 $\mathrm{km}$ year $^{-1}$, we observed the extinction of the metapopulation before the end of the simulation. With increasing isocline rate shift, the extinction of both the S-allele and the total metapopulation occurred earlier in time. The level of neutral allelic variation showed a sharp decline in the newly established populations (not shown) under all temperature increase scenarios.

An increase in the temperature variability resulted in a relative increase in the numbers of the generalist genotype GG compared with the numbers of specialists SS (Fig. 3). Under range shift, this relatively larger fraction of the G-allele and/or the relative fitness benefit of the G-allele under larger temperature variability led to an earlier extinction of the specialist allele $S$ under range shifts (Fig. 3). The differences in distributions of the three genotypes in time under range shifts between the levels of temperature variability were similar under all temperature increase scenarios.

\section{Effects of changing genotype distribution on species' persistence}

If the metapopulation was parameterized to contain only S-alleles (S-metapopulation) or G-alleles (G-metapopulation), its initial size was smaller than when both alleles S and G were present (SG-metapopulation) (Fig. 4). Under all parameter settings, the metapopulation size decreased during a range shift, and the decline of the metapopulation was steepest when it contained both alleles S and G. Compared at a set moment in time, the size of the SG-metapopulation was smaller than both the S- and the G-metapopulation sizes under the temperature increase scenario of $2 \mathrm{~km} \mathrm{year}^{-1}$. Under the range shifts induced by the $4 \mathrm{~km} \mathrm{year}^{-1}$ and the $8 \mathrm{~km}$ year $^{-1}$ scenario, the SG-metapopulation size became smaller than the G-metapopulation, but stayed larger than the S-metapopulation. As a result of the gradual decline of the S-allele frequency in the SG-metapopulation under range shift, the sizes of the SG- and G-metapopulations eventually converged under all temperature increase scenarios.

\section{Recovery of the original genotype distribution after temperature stabilization}

Our model simulations were continued after the implemented temperature increase stopped. In this period
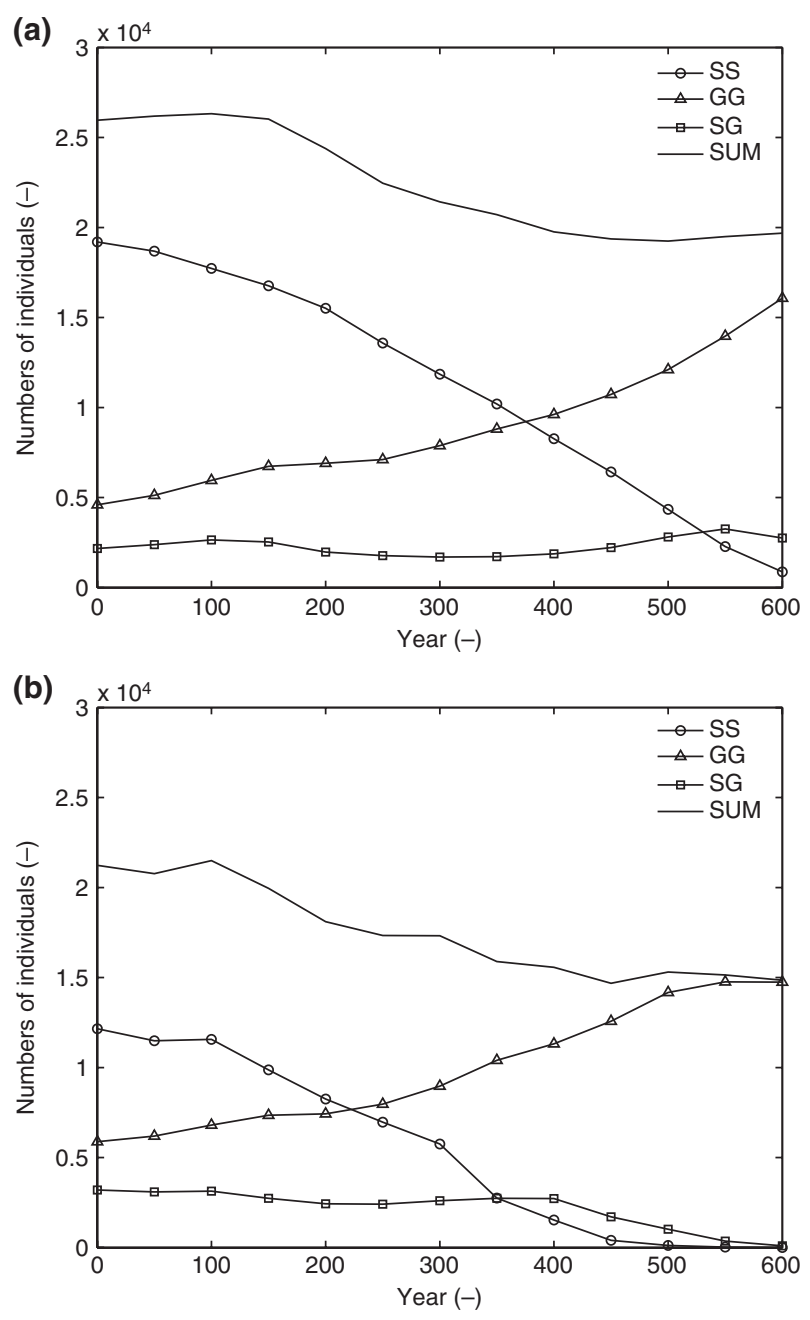

Fig. 3 The number of generalists (GG), specialists (SS), heterozygotes (SG) and their sum in time under the temperature increase scenario of $2 \mathrm{~km}$ year ${ }^{-1}$ for standard deviations of $0 \mathrm{~km}(\mathrm{a})$ and $280 \mathrm{~km}(\mathrm{~b})$.

the species' range still shifted, until the moment that the metapopulation was distributed evenly again around the temperature optimum. The initial, equilibrium distribution of the SS, SG and GG genotypes in the metapopulation could re-establish itself in the three following situations, which could also occur together:

1 If individuals carrying an S-allele were still present in the area where they have a selective advantage over the individuals with the GG genotype (Fig. 5 shows an example). In this case, the number of SS and SG individuals increased and they moved northwards until they took their original positions around the temperature optimum. Especially in the $2 \mathrm{~km}$ year $^{-1}$ scenario, recovery was often possible, even after long periods of temperature increase. However, the recovery period always took longer than the period of temperature increase (Fig. 5). 

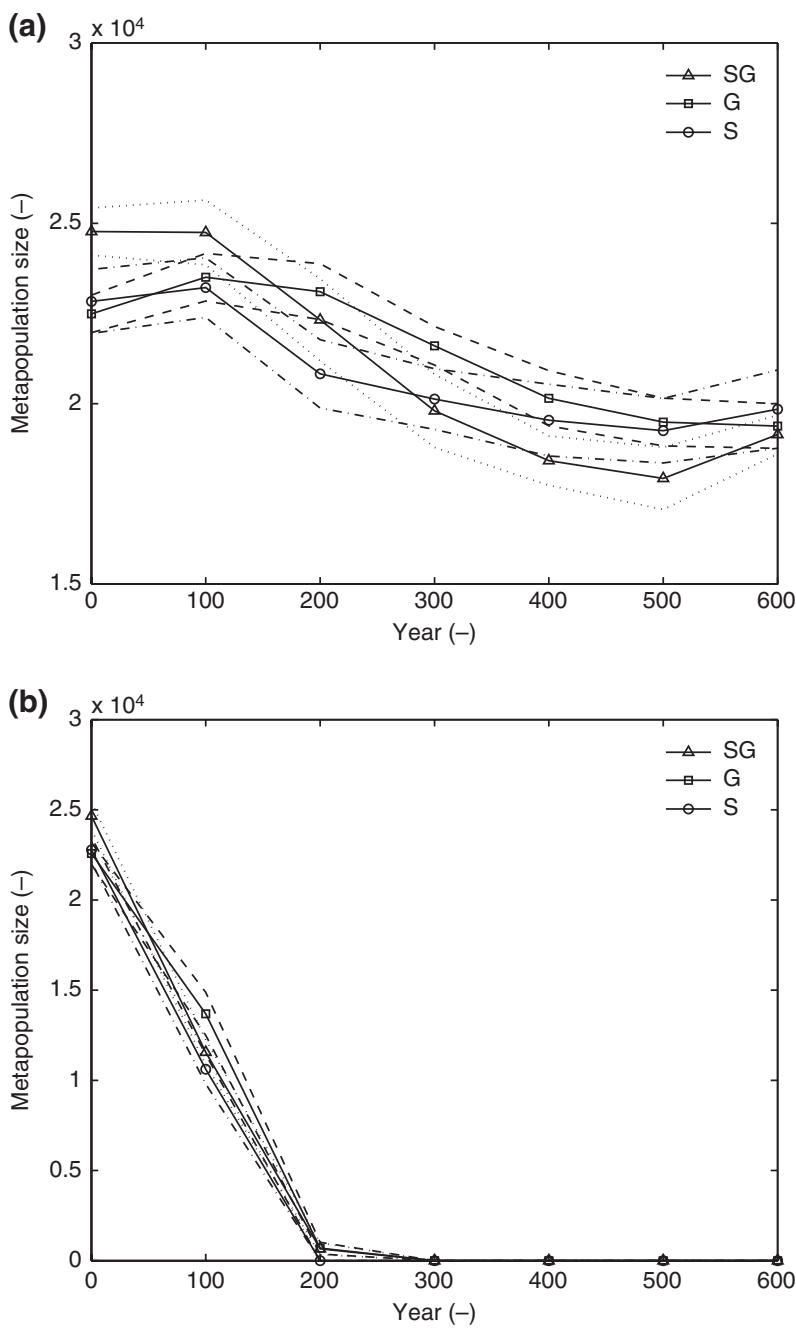

Fig. 4 The average size, and its $95 \mathrm{f}$ confidence interval, of the metapopulation, which consisted of only the generalist allele (G), of only the specialist allele (S) and of both alleles (SG), under the temperature increase scenario of $2 \mathrm{~km} \mathrm{year}^{-1}$ (a) and $8 \mathrm{~km} \mathrm{year}^{-1}$ (b). Note that the y-axes scales differ.

2 If a G-allele in the region around the temperature optimum mutated into an S-allele. In this situation, the individual with this SG-genotype had a selective advantage over the surrounding generalists. Once this mutation established itself, it led to an increase in the numbers of individuals with the SG- and SSgenotypes, until they took their original positions around the temperature optimum. Such a mutation occurred regularly (in 11 of 140 runs, for 14 parameter sets) and increased the distribution recovery rate, depending on the time and location of the mutation, in comparison with the rate without such a beneficial mutation.

3 In the exceptional case of a G-allele mutating into an S-allele at the leading edge of the metapopulation during the range shift. In the single example that we detected in our runs (Figure S4 in the Supporting Information), this mutation led to the persistence of the S-allele near the temperature optimum throughout the range shift. In this case, the relative increase in the generalist numbers was limited under range shift.

\section{Discussion}

The purpose of this study was twofold. First, to explore the interaction of local adaptation and founder events under range shifts in response to climate change and second, to determine the effects of this interaction on genotype distribution and species' persistence. We found that the equilibrium distribution of the genotypes in the species' range changed when the range was shifting under temperature increase. This change was caused by a founder effect. As a result, the genotypes ended up in geographical regions that differed from those regions in which they had the highest fitness. This led to a decreased habitat occupancy causing a decreased metapopulation size and, as such, this impeded the species' persistence. It furthermore caused the extinction of one of the genotypes, which survival time was further diminished under increased temperature variability.

The results of this study suggest that founder events that occur during range shift may have adverse consequences for a species' persistence. This comes about through the impeded local adaptation in newly established populations when these, at a later stage, become located closer to the species' range centre. Travis et al. (2007) showed the possibility of the establishment of deleterious mutations through founder events at expanding range margins. Here, we extend their work and show that the enhanced frequency, due to founder events, of the locally bestadapted genotype at the expanding range margin in time may also have adverse effects on the level of local adaptation throughout the species' range. In our model, this occurred despite the fact that genotypes better-adapted to the range centre were present in the species' range throughout the temperature increase and recovery period.

It has been suggested that local adaptation and range shifts could complement each other, leading to increased species' persistence under climate change (Hoffmann \& Sgro, 2011; Parmesan, 2006). Our study did not provide evidence supporting this suggestion. Instead, we showed that range shifts may lead to local maladaptation throughout the species' range and cause decreased species' persistence. 
The combined effect of range shift and local adaptation on the genotype distribution

As the location of the optimal temperature moved northwards, the suitable climate space for the individuals at the northern range margin of the metapopulation extended northwards as well. The newly available habitat patches became occupied by the generalists living at this northern range edge. In the northern transition area, the changing temperature conditions allowed the individuals carrying the specialist allele $S$ to expand their range as well. However, the expansion of the specialist genotype here was delayed. The observed delay was caused by the absence of the S-allele in the populations where this allele had the selective advantage (as indicated by Figure S4 in the Supporting Information). This resulted from founder events, the occurrence of which was confirmed by the patterns in the neutral allelic variation. So the habitat sites to which the specialist genotype needed to disperse were already occupied by the climate generalists and, due to their local dominance, most of the dispersers into these populations were generalists too. As a result, the establishment rate of the S-allele in these populations to the north of their distribution was too low to track the expansion of the generalist populations. Overall, the frequency of the Sallele in populations in the transition region increased, but more slowly than the expansion of the generalist range at the northern border of the species' range. Consequently, the size of the northern region where the generalists lived became constantly larger in time, and upon continuing range shift, the specialists ended up at the lagging range margin, where generalists were better-adapted to the changed climate and therefore performed better. So in this location, the specialist/ intermediate populations started to decrease as a result of selection for the better-adapted generalists.

An increased yearly temperature variability led to a further advantage for the climate generalists because its main effect was a decrease in size of the central part of the range in which the climate specialists could outcompete the individuals carrying a G-allele, so that the initial numbers of climate specialists were lower.

\section{The effect of the changing genotype distribution on the survival of the species}

To gain insight into the consequences of the described changes in species' persistence, we compared the size of the metapopulation when it had a mixed allele composition (containing both S- and G-alleles) with its size when it contained only either the S- or G-allele.

The initial size of the metapopulation was largest when it contained both alleles. This was presumably the result of the combined local benefits of the present genotypes, overall leading to larger population sizes. Under all parameter settings, the metapopulation size decreased during a range shift. This resulted from the inability to utilize the full potential range to the north of the temperature optimum because it took too long to establish new populations in the northern range that became habitable due to a fast shift of the temperature optimum (Schippers et al., 2011), even under the slowest temperature increase scenario. However, when the metapopulation contained both alleles $S$ and $G$ it declined most steeply. Under these settings, both the specialist and generalist genotypes ended up living under suboptimal climate conditions, which led to

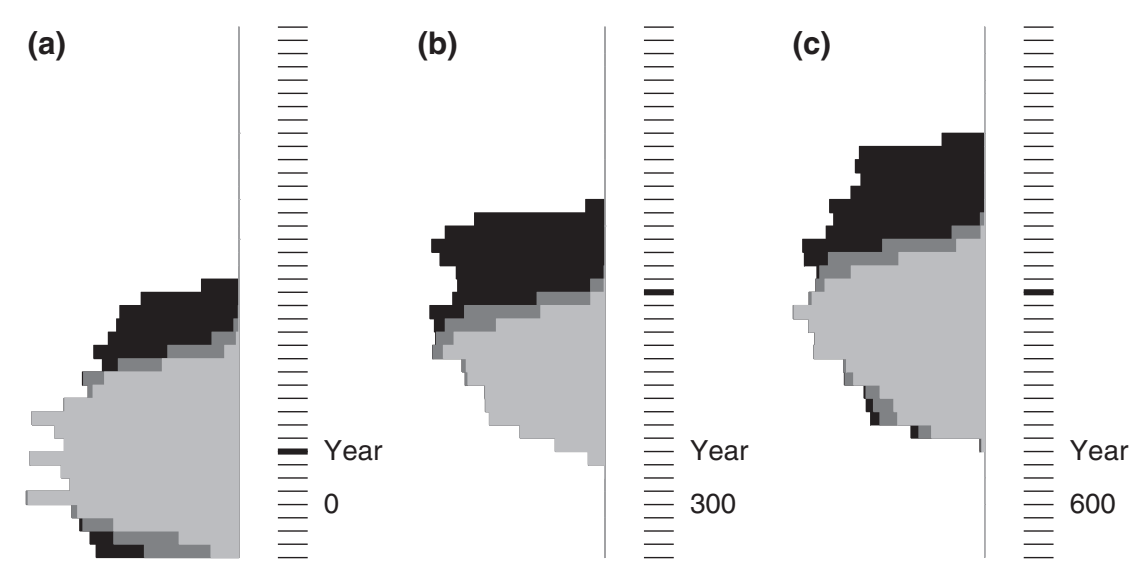

Fig. 5 The distributions and numbers of the GG (black bars), SG (dark grey bars) and SS (light grey bars) genotypes in time through the model space under the temperature isocline shift rate of $2 \mathrm{~km}$ year $^{-1}$, with standard deviation of $140 \mathrm{~km}$. The lengths of the bars indicate the sum of the local numbers of individuals. The thin horizontal lines represent the model space, cut into ranges of $50 \mathrm{~km}$. The bold black lines in the model space indicate the locations of the average temperature optimum along the total range of $2000 \mathrm{~km}$ in time, which is indicated in the lower right corners of each of the figures. The temperature increase was stopped after 300 years. After this the simulation continued for another 300 years. 
additional loss of individuals in this metapopulation. Counter-intuitively, the decrease of the metapopulation size was only stopped once the specialist allele $S$ went extinct, and the generalists could increase their numbers in the southern part of the species' range.

\section{Recovering the equilibrium genotype distribution after temperature stabilization}

The genetic structure resulting from founder effects under range expansion can be very persistent (Hewitt, 1996). Here, we showed for adaptive genes that the prolonged absence of locally beneficial alleles as a result of founder events, may lead to delayed local adaptation. However, local adaptation can be enhanced by beneficial mutations.

\section{Perspectives}

The rates of temperature increase we used were based on temperature change projections for the year 2100 and these levels were extrapolated over simulation periods of several hundreds of years. Increases in weather variability are projected for the next century, but difficult to quantify. We have therefore decided to explore the effect of changes in temperature variability by doubling the current standard deviation of the average temperature and by assuming a zero standard deviation. As a result we can state that the used rates of temperature increase in this study matched the projections for the next 100 years (Hadleycentre, 2003), whereas the ultimate temperatures after extrapolation and the incorporated levels of temperature variability served exploration purposes. The changes in genotype distributions were therefore only shown for the current standard deviation of the average temperature. In addition, we incorporated a model experiment to assess the distribution restoration time after a future moment of temperature stabilization.

The landscape used in this model is $15 \times 2000 \mathrm{~km}$, with a model species' maximum dispersal distance of $15 \mathrm{~km}$. Founder events are more likely to occur in linear habitats. However, the east and west sides of the used landscape were merged to avoid edge effects, which effectively created an infinitely wide landscape. In addition, founder events have been shown to take place under range shifts in response to contemporary climate change (Garroway et al., 2011). In this study, we show how such founder events can affect the distribution of genotypes across a species' range for a moderate disperser living in fragmented habitat. Bronnenhuber et al. (2011) showed in an empirical study that in an invasive fish species founder effects at the expansion front were mitigated through a dual-dispersal strategy which com- bines both long-distance and contiguous dispersal. This raises the question how species' ecology and the configuration of their habitat may affect the results found in this study and whether our results can be observed in empirical studies as well. Nolte (2011) therefore argued for a broader scope of empirical studies with Bronnenhuber et al. (2011) as an example.

We have assumed a random dispersal direction for our model species. Clobert et al. (2009) show that there is accumulating evidence that habitat selection is nonrandom for species in various taxa. Informed dispersal may influence the model results by increasing population growth rates. This could lead to a better tracking of the shifting temperature optimum and therefore increase species survival times. However, in other simulations (not published) we observed that increased numbers of immigrants do not significantly change the effect of founder events. Thus, the patterns in the spatial distribution of the various genotypes and their effects on metapopulation survival probabilities are likely not affected by our assumption of random dispersal.

We modelled genetic differences between central and marginal populations of this species' range. As still little is known about the basis and extent of genetic differentiation between central and marginal populations along a biogeographical gradient, our genetically simple model provides valuable insights into how founder effects under range shifts could interact with local adaptation in species' ranges along such a gradient. Here, we elaborate on the possible effects of several aspects that have not been included in the genetic model on the model results.

1 The number of occasions in which mutations affect the observed distribution patterns may be an overestimation due to the use of a two-allele gene in contrast to an infinite allele model. A lower mutation rate would not change the process of founder effects during colonization at the northern edge.

2 In this study, we have modelled a complex trait as $100 \%$ heritable. Although this is unrealistic, the results give an indication on how the interaction of range shifts and adaptation may affect the heritable part of important species traits.

3 We have omitted the possibility of mutations leading to an overall increased temperature tolerance of the species. Such mutations would likely, locally, replace the existing genotypes and increase the survival of the species. We have not included such mutations based on Parmesan (2006), who concludes in a review paper that 'there is no evidence for change in the absolute climate tolerances of a species', despite the occurrence of local evolutionary responses to climate change. 
The examples of mutation establishment that we observed in our results (see Figure S4 in the Supporting Information) suggest that the dominance of the GG genotype under range shift might not occur if the initial marginal populations would be genetically more diverse. The chance of a genotype to be enhanced by the founder effect is dependent of its relative fitness (Muenkemueller et al., 2011) and its frequency at the ancestral populations. The inclusion of a higher level of polymorphism or a smaller trait heritability in the model may lead to genetically more diverse populations in the range margin. A higher level of genetic diversity at the range margin could as well be generated by habitat heterogeneity, which was not included in our model. If this would prevent founder effects, such a management strategy may be designed to support local adaptation under climate change. To assess whether such a strategy is promising, additional modelling and empirical experiments are needed.

\section{Acknowledgements}

This research was supported by the Netherlands' Ministry of Agriculture, Nature Conservation and Food Quality through its strategic research programme: "Sustainable spatial development of ecosystems, landscapes, seas and regions" (projects KB-01-007-001, KB-01-007-013, KB-14-002-009) and by the Netherlands' National Research Programme Climate changes Spatial Planning (http://www.klimaatvoorruimte.nl/) as project A2.4.

\section{References}

Bronnenhuber JE, Dufour BA, Higgs DM, Heath DD (2011) Dispersal strategies, secondary range expansion and invasion genetics of the nonindigenous round goby, Neogobius melanostomus, in Great Lakes tributaries. Molecular Ecology, 20, 1845-1859.

Chen IC, Hill JK, Ohlemueller R, Roy DB, Thomas CD (2011) Rapid range shifts of species associated with high levels of climate warming. Science, 333, 1024-1026.

Clobert J, Le Galliard J-F, Cote J, Meylan S, Massot M (2009) Informed dispersal, heterogeneity in animal dispersal syndromes and the dynamics of spatially structured populations. Ecology Letters, 12, 197-209.

Cobben MMP, Verboom J, Opdam P, Hoekstra RF, Jochem R, Arens P, Smulders MJM (2011) Projected climate change causes loss and redistribution of genetic diversity in a model metapopulation of a medium-good disperser. Ecography, 34, 920-932.

Cobben MMP, Verboom J, Opdam PFM, Hoekstra RF, Jochem R, Smulders MJM (2012) Landscape prerequisites for the survival of a modelled metapopulation and its neutral genetic diversity are affected by climate change. Landscape Ecology, 27, 227-237.

Edmonds CA, Lillie AS, Cavalli-Sforza LL (2004) Mutations arising in the wave front of an expanding population. Proceedings of the National Academy of Sciences of the United States of America, 101, 975-979.

Excoffier L, Foll M, Petit RJ (2009) Genetic consequences of range expansions. Annual Review of Ecology Evolution and Systematics, 40, 481-501.

Garroway CJ, Bowman J, Holloway GL, Malcolm JR, Wilson PJ (2011) The genetic signature of rapid range expansion by flying squirrels in response to contemporary climate warming. Global Change Biology, 17, 1760-1769.
Hadleycentre (2003) Climate change: observations and predictions. Recent research on climate change science from the Hadley Centre. Hadley Centre for Climate Prediction and Research Department for Environment, Food and Rural Affairs Met Office, Exeter, UK, p. 12.

Hampe A, Petit RJ (2005) Conserving biodiversity under climate change: The rear edge matters. Ecology Letters, 8, 461-467.

Hewitt GM (1996) Some genetic consequences of ice ages, and their role in divergence and speciation. Biological Journal of the Linnean Society, 58, 247-276.

Hill JK, Thomas CD, Blakeley DS (1999) Evolution of flight morphology in a butterfly that has recently expanded its geographic range. Oecologia, 121, 165-170.

Hill JK, Griffiths HM, Thomas CD (2011) Climate change and evolutionary adaptations at species' range margins. Annual Review of Entomology, 56, 143-159.

Hoffmann AA, Sgro CM (2011) Climate change and evolutionary adaptation. Nature, 470, 479-485.

IPCC (2007) IPCC Fourth Assessment Report (AR4) Climate Change 2007: Synthesis Report Contribution of Working Groups I, II and III to the Fourth Assessment Report of the Intergovernmental Panel on Climate Change Core Writing Team (eds. Pachauri RK Reisinger A), Switzerland, IPCC, Geneva, Switzerland. p. 104

Jump AS, Hunt JM, Martinez-Izquierdo JA, Penuelas J (2006) Natural selection and climate change: temperature-linked spatial and temporal trends in gene frequency in Fagus sylvatica. Molecular Ecology, 15, 3469-3480.

Kawecki TJ (2008) Adaptation to marginal habitats. Annual Review of Ecology Evolution and Systematics, 39, 321-342.

Klopfstein S, Currat M, Excoffier L (2006) The fate of mutations surfing on the wave of a range expansion. Molecular Biology and Evolution, 23, 482-490.

Lee CE (2002) Evolutionary genetics of invasive species. Trends in Ecology \& Evolution, 17, 386-391.

Mayr E (1942) Systematics and the origin of species. Columbia University Press, New York.

McInerny GJ, Turner JRG, Wong HY, Travis JMJ, Benton TG (2009) How range shifts induced by climate change affect neutral evolution. Proceedings of the Royal Society B-Biological Sciences, 276, 1527-1534.

Muenkemueller T, Travis JMJ, Burton OJ, Schiffers K, Johst K (2011) Density-regulated population dynamics and conditional dispersal alter the fate of mutations occurring at the front of an expanding population. Heredity, 106, 678-689.

Nachman MW, Crowell SL (2000) Estimate of the mutation rate per nucleotide in humans. Genetics, 156, 297-304.

Nolte AW (2011) Dispersal in the course of an invasion. Molecular Ecology, 20, 1803-1804.

Parmesan C (2006) Ecological and evolutionary responses to recent climate change. Annual Review of Ecology Evolution and Systematics, 37, 637-669.

Parmesan C, Yohe G (2003) A globally coherent fingerprint of climate change impacts across natural systems. Nature, 421, 37-42.

Root TL, Price JT, Hall KR, Schneider SH, Rosenzweig C, Pounds JA (2003) Fingerprints of global warming on wild animals and plants. Nature, 421, 57-60.

Schippers P, Verboom J, Vos CC, Jochem R (2011) Metapopulation shift and survival of woodland birds under climate change: will species be able to track? Ecography, 34, 909-919.

Sexton JP, Mcintyre PJ, Angert AL, Rice KJ (2009) Evolution and ecology of species range limits. Annual Review of Ecology Evolution and Systematics, 40, 415 436

Thomas CD, Bodsworth EJ, Wilson RJ, Simmons AD, Davies ZG, Musche M, Conradt L (2001) Ecological and evolutionary processes at expanding range margins. Nature, 411, 577-581.

Travis JMJ, Muenkemueller T, Burton OJ, Best A, Dytham C, Johst K (2007) Deleterious mutations can surf to high densities on the wave front of an expanding population. Molecular Biology and Evolution, 24, 2334-2343.

Verboom J, Foppen R, Chardon P, Opdam P, Luttikhuizen P (2001) Introducing the key patch approach for habitat networks with persistent populations: an example for marshland birds. Biological Conservation, 100, 89-101.

Vila C, Sundqvist AK, Flagstad O et al. (2003) Rescue of a severely bottlenecked wolf (Canis lupus) population by a single immigrant. Proceedings of the Royal Society of London Series B-Biological Sciences, 270, 91-97.

Vos CC, Verboom J, Opdam PFM, Ter Braak CJF (2001) Toward ecologically scaled landscape indices. American Naturalist, 157, $24-41$. 


\section{Supporting Information}

Additional Supporting Information may be found in the online version of this article:

Appendix S1. Appendix with detailed model description and Figure S4.

Figure S1. The average survival probabilities of the SS (specialist) and GG (generalist) individuals, relative to the location of the temperature optimum, resulting from their perceived habitat qualities (see Fig. 1).

Figure S2. The average numbers of offspring of the SS (specialist) and GG (generalist) females, relative to the location of the temperature optimum, resulting from their perceived habitat qualities (see Fig. 1).

Figure S3. The average dispersal rates of the SS (specialist) and GG (generalist) individuals, relative to the location of the temperature optimum, resulting from their perceived habitat qualities (see Fig. 1).

Figure S4. The distributions and numbers of the GG (black bars), SG (dark grey bars) and SS (light grey bars) genotypes in time through the model space under the temperature isocline shift rate of $4 \mathrm{~km} \mathrm{year}^{-1}$, with standard deviation of $140 \mathrm{~km}$. The lengths of the bars indicate the sum of the local numbers of individuals. The thin horizontal lines represent the model space, cut into ranges of $50 \mathrm{~km}$. The bold black lines in the model space indicate the locations of the average temperature optimum along the total range of $2000 \mathrm{~km}$ in time, which is indicated in the lower right corners of each of the figures. The temperature increase was stopped after 300 years. In this particular run, in year 100 there were several SG-individuals at the range front, indicated by the arrow. These could establish as a result of their selective advantage and their increase in numbers was subsequently enhanced by the founder effect, leading to high numbers of specialists and intermediates throughout the range.

Please note: Wiley-Blackwell are not responsible for the content or functionality of any supporting materials supplied by the authors. Any queries (other than missing material) should be directed to the corresponding author for the article. 